\title{
Guided Osteogenesis using Titanium Nickelide Mesh Constructs Implanted into a Bone Cavitary Defect
}

Dor. $10.17691 / \mathrm{stm} 2017.9 .2 .08$

Received April 8, 2016

(c)

Yu.M. Iryanov, DSc, Professor, Chief Researcher, Laboratory of Morphology;

O.V. Dyuryagina, PhD, Senior Researcher, Laboratory of Purulent Osteology and Limb Defect Filling

Russian llizarov Scientific Center for Restorative Traumatology and Orthopedics, 6 M. Ulyanova St., Kurgan, 640014, Russian Federation

The aim of the investigation was to study the morphological characteristics of reparative osteogenesis using titanium nickelide mesh constructs implanted into a cavitary defect of the rat femoral metaphysis.

Materials and Methods. Reparative bone formation after implantation of titanium nickelide mesh structures into a cavitary defect of femoral metaphysis has been studied experimentally on rats. The following methods were used in the work: radiography, light and scanning electron microscopy, electron probe X-ray microanalysis.

Results. The implant microporous surface has been established to provide osteoblast adhesion, as well as osseointegration and adsorption of endogenic bone morphogenetic proteins. The implant possesses osteoconductive and osteoinductive characteristics, stops inflammatory processes. The membrane protective barrier, which prevents connective tissue ingrowth and provides guided osteogenesis, has formed around the implant in the defect periosteal zone. The defect is replaced with cancellous bone, the volumetric density of which is 1.5 times more than the control values.

Key words: bone cavitary defect; guided reparative osteogenesis; mesh constructs from titanium nickelide; implantation.

The main factor hindering the restitution of largevolume bone defects is invasion of the connective tissue from the periosteal surface due to a greater migration speed of fibroblasts compared to osteogenic cells [1-3]. It can inhibit completely or partially the process of reparative bone formation and be a cause of defect replacement with a dense scar-like connective tissue. To provide optimal conditions for forming organotypic regenerate, a technique of guided reparative osteogenesis using membrane technology preventing the connective tissue spreading has been developed $[4,5]$. Membranes from synthetic and natural materials are used for this purpose, though they are not osseointegrating, may induce inflammatory reaction and tissue edema, besides a repeated operative intervention is often required [6, 7]. New opportunities appeared owing to the introduction of medical technologies associated with the application of implants based on nickel and titanium, whose mechanical characteristics are very close to those of the bone tissue and are biocompatible [8-10]. However, this material was investigated only on the models of small bone defects (holes) without a control series of experiments. The efficiency of treating large cavitary bone defects by implanting titanium nickelide mesh and the process of bone regenerate formation have not yet been studied. It signifies the importance of the problem and the necessity of conducting further investigations.

The aim of the investigation was to study the morphological characteristics of reparative osteogenesis using titanium nickelide mesh constructs implanted into a cavitary defect of the rat femoral metaphysis.

Materials and Methods. Cavitary defects of femoral metaphysis were modeled on adult Wistar males in the experimental $(n=20)$ and control $(n=20)$ groups. The defect volume amounted to about $40 \%$ of the total metaphysis volume. Implants were placed to the animals of the experimental group, whereas in the control group no additional manipulations were performed. The work was performed in accordance with ethical principles

For contacts: Yuriy M. Iryanov, e-mail: irianov@mail.ru 

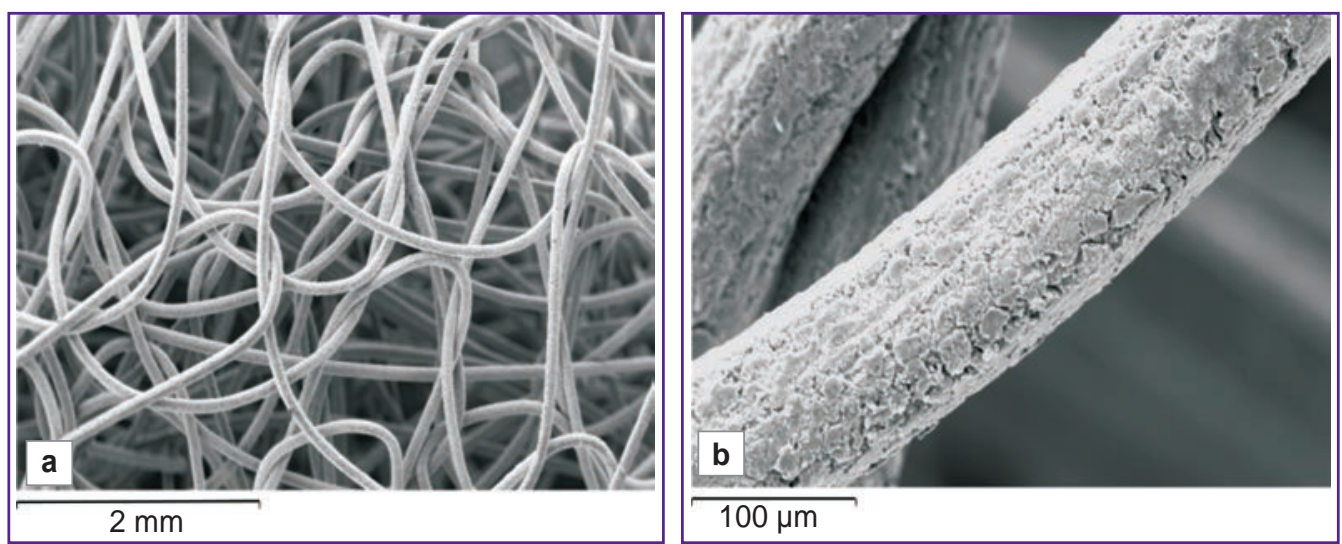

Figure 1. Implant appearance (a), and a microrelief of the implant filament surface layer (b); scanning electron microscopy; (a) $\times 20$; (b) $\times 180$

established by European Convention for the Protection of Vertebrata used for Experimental and other Scientific Purposes (the Convention was passed in Strasbourg, March 18, 1986, adopted in Strasbourg, June 15, 2006), and in compliance with the order of the Ministry of Health and Social Development of the Russian Federation No.708H of 23 August, 2010 "On the approval of laboratory practice rules". The study was approved by Ethics Committee of the Russian Ilizarov Scientific Center for Restorative Traumatology and Orthopedics.

The implant represented a mesh scaffold made from grade $\mathrm{TH}-10,90 \mu \mathrm{m}$ thick titanium nickelide filament, formed like a knitted fabric with open-ended macropores of 100-300 $\mu \mathrm{m}$ in diameter (Figure 1 (a)) [11].

The implant filament was fabricated from the composite material, its core consisted of nanostructural monolithic titanium nickelide, and microporous surface layer (Figure 1 (b)) 5-7 $\mu$ m thick was from titanium oxide (Scientific Production Enterprise MITS of Research Institute of Medical Materials and Shape Memory Implants, Siberian Physical-Technical Institute, Russia, Certificate No.POOCRU.AЯ79H18304). At 7, 14, 30, and 60 days the animals were withdrawn from the experiment (5 animals at each time period). Meta-epiphyseal zone of the bone was sawn out, fixed in $2 \%$ solution of paraformaldehyde and glutaraldehyde in $0.1 \mathrm{M}$ sodium phosphate buffer at $\mathrm{pH}=7.4$, dehydrated and imbedded in araldite and paraffin (after decalcification). Histological sections were stained with hematoxylin and eosin, and according to Van Gieson method. The araldite blocks were examined using INCA-200 Energy electron probe X-ray microanalyzer (Oxford Instruments, UK) and JSM840 scanning electron microscope (Jeol, Japan). Bone tissue volume density, compactness index and content of calcium, phosphorus, magnesium, sodium, and sulfur were determined in the regenerate with the help of these devices [8].

Statistical analysis was done using Microsoft Excel 2010 program. The data were presented as mean $(M)$, standard error of mean $(m)$, and significance level $(p)$.
The significance of intergroup differences in the compared parameters, given the distribution is normal, was calculated using Student's t-test. Differences were considered statistically significant at $p<0.05$.

Results and Discussion. 7 and 14 days after the operation signs of bone formation process, which was going on from the periosteum, endosteum, bone marrow, and the damaged bone structures of the defect edges, were noted in both animal groups.

In the control group of animals, a connective tissue regenerate was being formed in the periosteal zone, the band-shaped collagen fibers of which grew from the periosteum of the unaffected bone areas into the defect. The central defect zone was replaced with a loose connective and granulation tissue, in which foci of lymphocytic and plasmacytic infiltration were located, exudates, cellular detritus and fibrin bunches were detected. At the outer defect edges in the area of the unaffected periosteum as well as from the endosteum, islets of reticulofibrous bone tissue were being formed represented by the fine-meshed network of twisted bone-osteoid trabeculae of various maturation degree. At the inner defect edges, there were randomly positioned fragments of the injured bone structures, generated during defect modeling, and also separate isolated foci of a newly formed bone tissue in the form of short boneosteoid trabeculae lining the internal defect surface.

Quantitative investigations were carried out after the subsidence of the inflammatory processes by the end of the second week. The bone tissue volume density in the defect and regenerate compactness index were established to be equal to about $30 \%(p<0.001)$ of the values of the unaffected metaphysis 14 days after the operation in the group of control animals (Table 1).

A thin membrane-like envelope from irregular connective tissue formed by wavelike flat bundles of tightly interwoven collagen fibers oriented in various directions was formed 7 and 14 days after the operation in the periosteal defect zone around the implant filaments and cells in the experimental animal group 


\section{BIOMEDICAL INVESTIGATIONS}

Table 1

Characteristic of the bone tissue in the metaphysis cavitary defect in the control and experimental groups of animals and in the intact contralateral limb metaphysis $(\mathrm{M} \pm \mathrm{m})$

\begin{tabular}{|c|c|c|c|c|c|c|c|}
\hline \multirow{3}{*}{ Indices } & \multicolumn{6}{|c|}{ Experiment duration (days) } & \multirow{3}{*}{$\begin{array}{c}\text { Intact } \\
\text { metaphysis }\end{array}$} \\
\hline & \multicolumn{2}{|c|}{14} & \multicolumn{2}{|c|}{30} & \multicolumn{2}{|c|}{60} & \\
\hline & Control & Experiment & Control & Experiment & Control & Experiment & \\
\hline Bone tissue volume density (\%) & $8.44 \pm 0.38$ & $12.17 \pm 0.61^{*}$ & $13.54 \pm 0.68$ & $19.76 \pm 0.96^{*}$ & $15.02 \pm 0.74$ & $22.98 \pm 1.09 *$ & $25.07 \pm 1.21$ \\
\hline Compactness index & $0.09 \pm 0.01$ & $0.14 \pm 0.01^{* *}$ & $0.16 \pm 0.01$ & $0.25 \pm 0.02 * *$ & $0.18 \pm 0.01$ & $0.30 \pm 0.02^{* *}$ & $0.33 \pm 0.02$ \\
\hline
\end{tabular}

$\mathrm{N}$ o t e. Statistical significance of intergroup differences: ${ }^{*} p<0.001 ;{ }^{* *} p<0.01$.
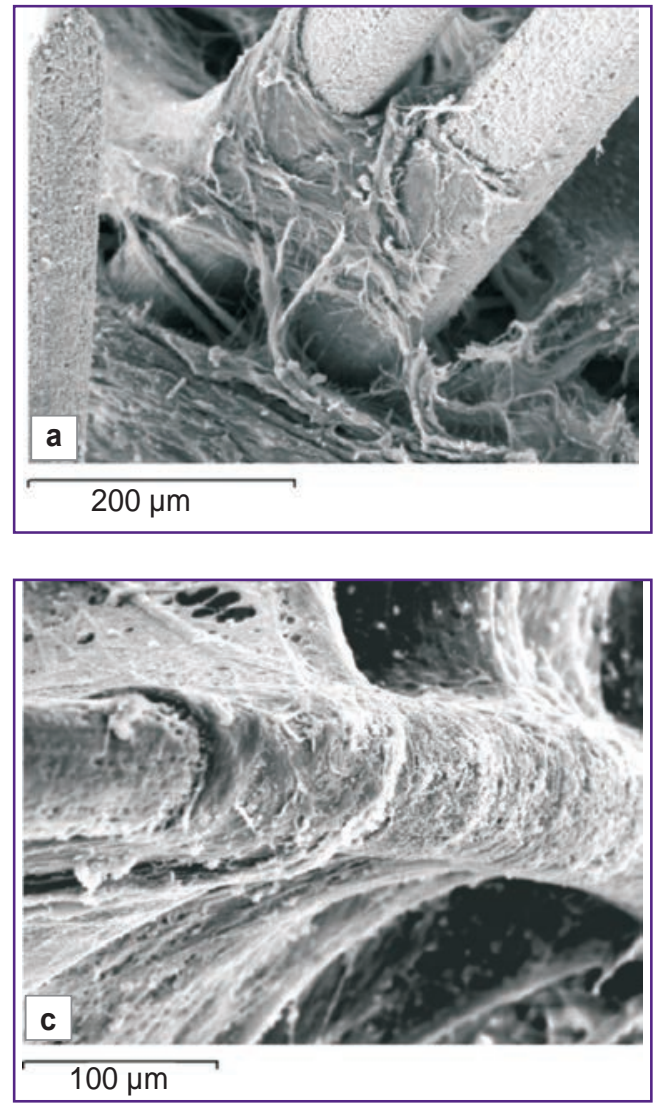

(Figure 2 (a)). The envelope started to form on the filament surface at the sites of its interlacements and propagated from periphery of the cells to their center. The envelope had a laminated structure. The inner layer was composed of a dense regular connective tissue. Collagen fibers looked like dense circularly oriented string-like bundles, which braided the implant filaments like a muff, were rigidly fixed to the filament microporous surface, grew in the gaps between them and provided fixation between implant filaments and in the bone defect (Figure 2 (b)). The outer layer was formed by a dense irregular connective tissue, whose thin plane bundles of collagen fibers were located with a fine-meshed network in different directions between the string-like bundles of the inner layer framework,

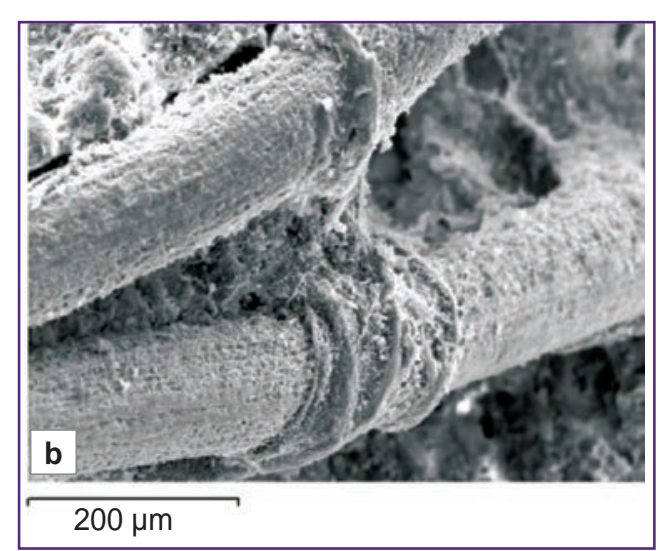

Figure 2. A connective tissue envelope on the surface of the implant structure in the periosteal defect area 7 (a) and 14 days (b), (c) after the operation; scanning electron microscopy, $\times 160$

and were connected with the surrounding bone and soft tissue structures (Figure 2 (c)). Under the connective tissue envelope in the endosteal and central zone of the defect and along its edges around the implant structures and on their surfaces, areas of active appositional bone formation were determined. A layer of the reticulofibrous bone tissue 300-400 $\mu \mathrm{m}$ thick was formed directly on the implant filament surface, generating a dense osseointegration (Figure 3 (a)). Implant filaments were covered with mineralizing bone matrix in the regions of osseointegration. Newly formed trabeculae grew into the fine-meshed implant structure (Figure 3 (b), (c)).

The results of quantitative investigations (See Table 1) showed a significant activation of the reparative osteogenesis and increase of maturation degree of the 


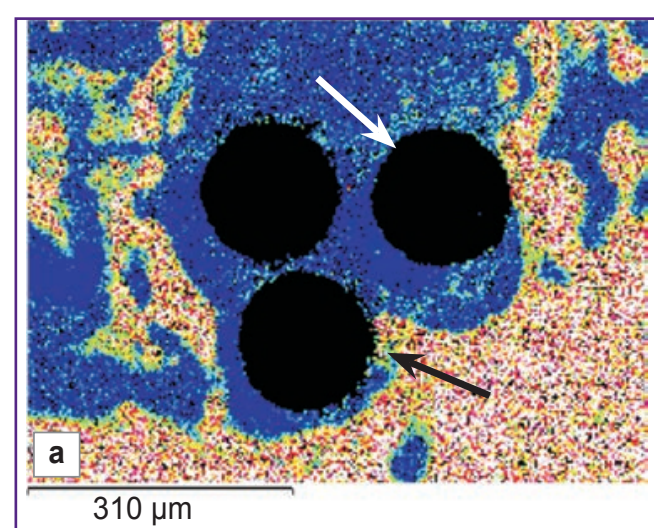

Figure 3. Guided reparative osteogenesis in the metaphysis defect 7 (a) and 14 days (b), (c) after the operation:

(a), (b) are the maps obtained by electron probe microanalysis, images are in the characteristic X-ray emission of calcium atoms; (c) scanning electron microscopy (organic components are removed with $6 \%$ solution of sodium hypochlorite); areas of osseointegration are shown by arrows; (a) $\times 100$; (b) $\times 25$; (c) $\times 70$

newly generated bone tissue in the animal regenerates of the experimental group compared to the control group. Thus, the volume density of the bone tissue in the defect was $144.19 \%$, and the compactness index $155.56 \%$ in comparison with the values in the control group $(p<0.001)$.

30-60 days after the operation, the defect in the control group was replaced by the regenerate with the prevalence of weakly mineralized dense irregular connective tissue growing from the periosteal surface (Figure 4 (a), (d)). The operated metaphysis zone acquired a marked cone-shaped form. A weakly calcified dense connective tissue prevailed in the regenerate periosteal zone. Fine-mesh bone structures of the periosteal regenerate merged with the newly generated endosteal trabeculae, grew arc-wise in the defect central zone, and formed a thin falciform layer of a newly generated cortical bone. Osteogenesis foci were observed in the central and marginal defect zones, where areas of osteoid and fragments of weakly mineralized reticulofibrous bone trabeculae isolated from each other by wide interlayers of a loose connective tissue with cavities filled with lymphocytic and macrophage elements were detected.

The bone tissue volume density in the defect, regenerate compactness index, calcium and phosphorus content made $50-60 \%$ of the intact metaphysis values $(p<0.001)$ (Tables 1 and 2$)$.

In the experimental groups $30-60$ days after the
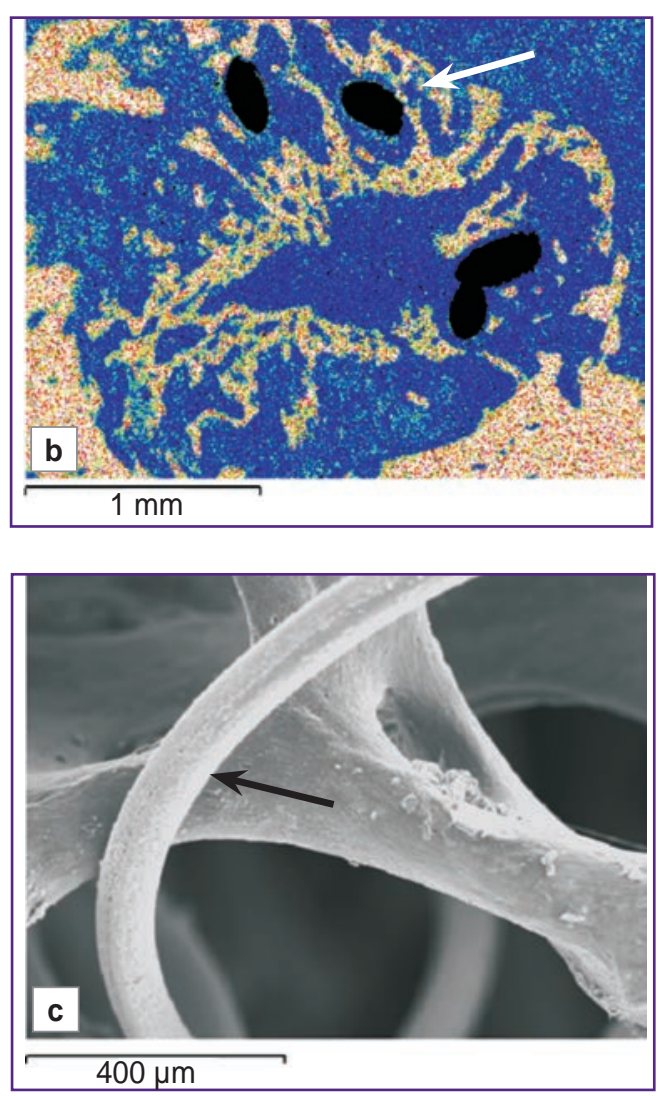

operation the bone defect area was replaced by the regenerate, in which cancellous bone prevailed (Figure 4 (b), (e)). A de novo formed area of cortical layer was presented by a compact bone with a lamellar structure. On the periosteal defect surface around the implant there were bundles of collagen fibers of the dense connective tissue of the protective envelope forming interlacements resembling village wicker fences (Figure 4 (c)). Implant filaments were surrounded with osteoid or overgrown completely with a newly generated bone tissue forming a composite, i.e. a compact bone reinforced with titanium nickelide (Figure 4 (f)). The bone tissue volume density, compactness index, and regenerate mineralization degree were slightly less compared to the intact metaphysis values, but by the end of the experiment these differences were not statistically significant, at the same time, they were more than 1.5 times greater $(p<0.001)$ than the indices in the control group (See Tables 1 and 2).

The results of the conducted investigation have shown that a layer of the dense connective tissue functioning as a biological protective barrier and preventing the ingrowth of paraosseous connective tissue is formed in the periosteal defect area on the implant surface. Besides, the defect is replaced with a cancellous bone, the volume density of which is more than 1.5 times higher than the control values at all stages of the experiment, and its mineral composition approximates the cancellous bone indices of the intact metaphysis by 

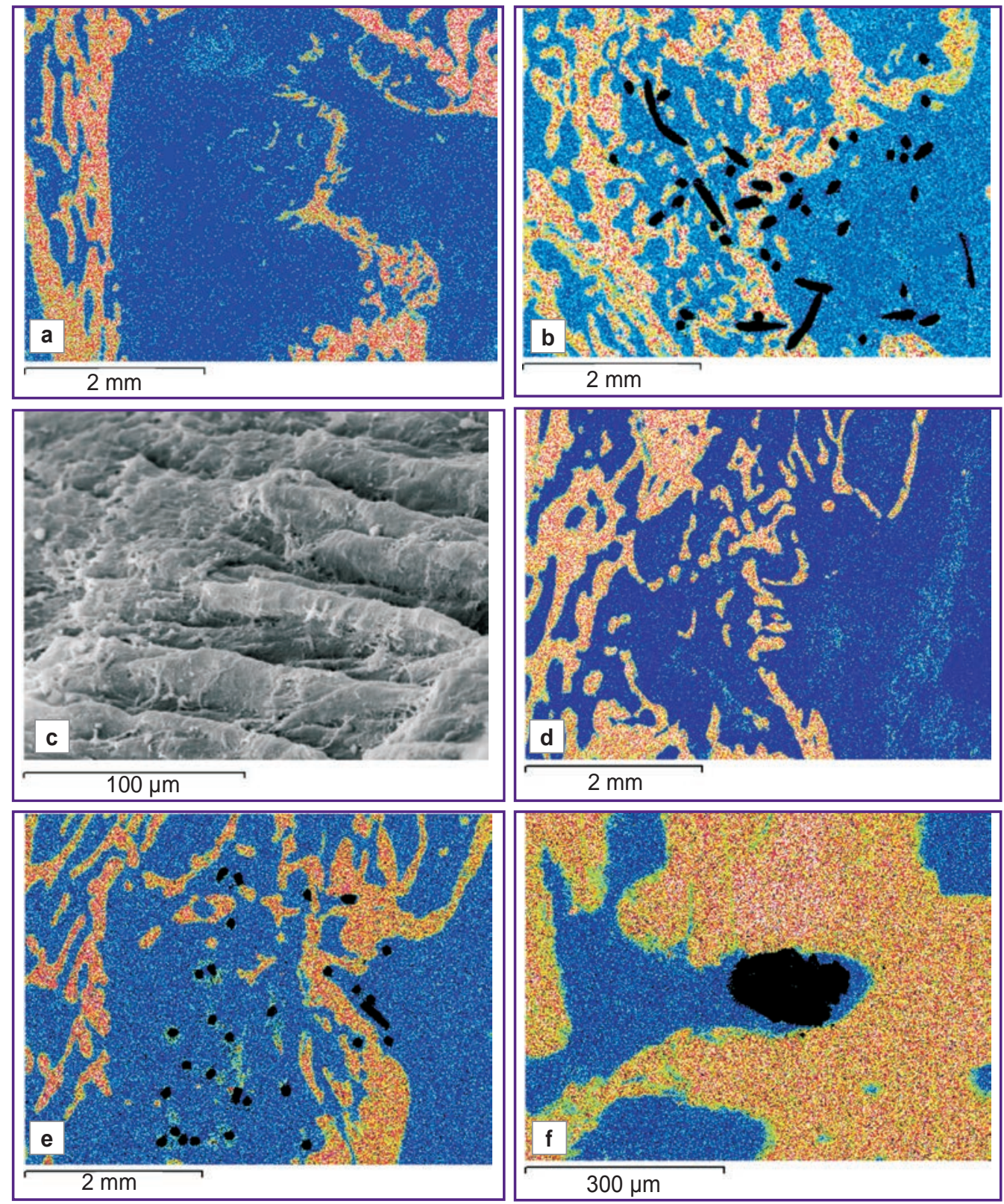

Figure 4. Replacement of metaphysis defect 30 (a)-(c) and 60 days (d)-(f) after the operation in the control (a), (d) and experimental (b), (c), (e), (f) groups of animals:

$(a),(b),(d)-(f)$ the maps obtained by electron probe microanalysis, images are in the characteristic X-ray emission of calcium atoms; (a), (b), (d), (e) $\times 20$; (f) $\times 100$; (c) scanning electron microscopy, $\times 500$

Table 2

Content of osteotropic chemical elements in the regenerate (\%) being formed in the metaphysis cavitary defect in the control and experimental animal groups 60 days after the operation, and in the intact metaphysis of the contralateral limb $(\mathrm{M} \pm \mathrm{m})$

\begin{tabular}{|lccc|}
\hline Elements & Control & Experiment & Intact metaphysis \\
\hline Sodium & $0.41 \pm 0.02$ & $0.44 \pm 0.02$ & $0.40 \pm 0.02$ \\
\hline Magnesium & $0.22 \pm 0.01$ & $0.25 \pm 0.01$ & $0.22 \pm 0.01$ \\
\hline Phosphorus & $1.93 \pm 0.04$ & $2.94 \pm 0.13^{*}$ & $3.22 \pm 0.15$ \\
\hline Sulfur & $0.23 \pm 0.01$ & $0.30 \pm 0.02^{* *}$ & $0.22 \pm 0.01$ \\
\hline Calcium & $3.85 \pm 0.16$ & $5.89 \pm 0.26^{*}$ & $6.44 \pm 0.31$ \\
\hline
\end{tabular}

$\mathrm{N}$ o t e. Statistical significance of intergroup differences: ${ }^{*} p<0.001 ;{ }^{* *} p<0.05$. 
the end of the experiment. Reparative bone formation is going on as a direct intramembrane and appositional osteogenesis. No cases of inflammatory process are observed, which confirms our previous data [8-10]. The microporous structure of the implant filament surface layer provides adhesion of osteoblasts, osseointegration and osteoinductive properties. Owing to the capillary properties of the implant, adsorption of endogenic bone morphogenetic proteins takes place. The functional activity of these proteins provides osteoinduction of the implant [12].

Conclusion. The implant from titanium nickelide mesh constructs possesses good biocompatibility, osteoconductive, osteoinductive, and osteoplastic characteristics, arrests inflammatory process, and can be successfully applied for replacement of large cavitary bone defects.

Study Funding. The study was performed within the framework of the approved project of the scientific research work.

Conflicts of Interest. The authors of this article have no conflicts of interest to disclose.

\section{References}

1. Liu J., Kerns D.G. Mechanisms of guided bone regeneration: a review. Open Dent J 2014; 8(1): 56-65, https:// doi.org/10.2174/1874210601408010056.

2. Kim J.-Y., Yang B.-E., Ahn J.-H., Park S.O., Shim H.-W. Comparable efficacy of silk fibroin with the collagen membranes for guided bone regeneration in rat calvarial defects. J Adv Prosthodont 2014; 6(6): 539, https://doi. org/10.4047/jap.2014.6.6.539.

3. Jung R.E., Fenner N., Hämmerle C.H.F., Zitzmann N.U. Long-term outcome of implants placed with guided bone regeneration (GBR) using resorbable and non-resorbable membranes after 12-14 years. Clin Oral Implants Res 2012;
24(10): 1065-107, https://doi.org/10.1111/j.1600-0501.2012. 02522.x.

4. Hammerle C.H.F., Jung R.E. Bone augmentation by means of barrier membranes. Periodontol 2000 2003; 33(1): 36-53, https://doi.org/10.1046/j.0906-6713.2003.03304.x.

5. Karring T., Nyman S., Gottlow J., Laurell L. Development of the biological concept of guided tissue regeneration animal and human studies. Periodontol 2000 1993; 1(1): 26-35, https://doi.org/10.1111/j.1600-0757.1993.tb00204.x.

6. Schmidmaier G., Baehr K., Mohr S., Kretschmar M., Beck S., Wildemann B. Biodegradable polylactide membranes for bone defect coverage: biocompatibility testing, radiological and histological evaluation in a sheep model. Clin Oral Implants Res 2006; 17(4): 439-444, https://doi.org/10.1111/ j.1600-0501.2005.01242.x.

7. van Leeuwen A.C., Huddleston Slater J.J.R., Gielkens P.F.M., de Jong J.R., Grijpma D.W., Bos R.R.M. Guided bone regeneration in rat mandibular defects using resorbable poly(trimethylene carbonate) barrier membranes. Acta Biomater 2012; 8(4): 1422-1429, https://doi.org/10.1016/j. actbio.2011.12.004.

8. Iryanov Yu.M., Iryanova T.Yu. Bone defect replacement under conditions of transosseous osteosynthesis and titanium nickelide implant application. Morfologiia 2012; 142(4): 83-86.

9. Iriyanov Y.M., Chernov V.F., Radchenko S.A., Chernov A.V. Plastic efficiency of different implants used for repair of soft and bone tissue defects. Bull Exp Biol Med 2013; 155(4): 518-521, https://doi.org/10.1007/s10517-013-2191-4.

10. Irianov I.M., Diuriagina O.V., Karaseva T.I., Karasev E.A. The osteoplastic effectiveness of the implants made of mesh titanium nickelide constructs. Bosn J Basic Med Sci 2014; 14(1): 4-7.

11. Iryanov Y.M., Iryanova T.Y. Implantat dlya zameshcheniya defekta kosti [The implant to replace bone defect]. RF Patent 111759, МПК: A61F2/28. 2011.

12. Uebersax L., Hagenmüller H., Hofmann S., Gruenblatt E., Müller R., Vunjaknovakovic G., Kaplan D.L., Merkle H.P., Meinel L. Effect of scaffold design on bone morphology in vitro. Tissue Eng 2006; 12(12): 3417-3429, https://doi.org/10.1089/ten.2006.12.3417. 